\title{
The IJSPP Twitter Account: Our Secondary Step to Narrow the Gap Between Sport Science and Sport Practice
}

One of the strengths of the International Journal of Sports Physiology and Performance (IJSPP) is the section of Practical Applications in each published article. This section is intended to address our mission to "promote the publication of research in sport physiology and related disciplines that has direct practical application to enhancing sport performance, preventing decrements in performance, or enhancing recovery of athletes." With the Practical Applications section, we try to narrow the often-mentioned gap between sport science and sport practice. ${ }^{1-3}$ By clear identification of authentic practical advice for athletes and practitioners, ${ }^{2}$ we are attempting to "sow the fields for later harvest."

However, just sowing the fields is not enough. A next step in narrowing the gap between science and practice is to actively deliver our message to the end users - the coaches - at the field, court, pool, or track. One obvious vehicle for this strategy is social media. It is clear that almost everybody is nowadays "connected" and that the amount of information spread on a variety of social media platforms is enormous. This challenges us to think of how IJSPP's message can stand out from the masses. That is not a complicated process. Just be the same as we always have been and, as is the case for the Practical Applications, deliver authentic practical advice to our readership.

To accomplish this, IJSPP has appointed a Social Media Editor, Teun van Erp, who will lead us, together with Associate Editors Rob Lamberts and Stephen Cheung and Editorial Assistant Dionne Noordhof, in this task. Teun has experience as a sport scientist and has also been involved, for more than 10 years, as a leading scientist with one of the major Union Cycliste Internationale WorldTour cycling teams. As such, he has a very keen eye on the application of science in the field and is very well equipped to lead this task.

For many scientists and also scientific journals, Twitter is the most popular platform to create social awareness about recently published papers and issues. Just like word of mouth is the best form of advertising, oftentimes the best notice for a paper comes from fellow scientists talking about the work. Posting can take many forms beyond a simple summary and link crammed into 280 characters. For some authors, it is also a creative pursuit, ranging from including key figures to infographics or image files summarizing the paper. Posting about papers is more than self-promotion. Instead, it is a venue for scientific communication to the end users.

As part of this activity, it is important for journals to have a high number of Twitter followers, as this increases the social impact of a journal's tweets. In return, the tweets are an easy way for scientists, coaches, and other people interested in sport science research to keep track of recently accepted or published papers. The number of Twitter followers of the top-15 scientific journals ranges from 837 to 76,300. Interestingly, there is a strong correlation $(r=.81)$ between a journal's number of Twitter followers and its impact factor (Figure 1). This highlights the fact that a scientific article in a journal not only needs to be of high quality but also needs social awareness in order to optimally share the body of knowledge with the end users.
As you may have noticed, IJSPP has since the beginning of October 2020 started to increase the social awareness of each published issue and newly posted ahead-of-print papers through Twitter (@IJSPPJournal).This initiative over October and November 2020 led to a monthly increase of overall tweet impressions $(>3000 \%)$ and new monthly Twitter followers $(+310 \%)$ compared with the first 9 months of 2020 .

Our plan is to create more awareness about articles that are published in IJSPP and those that have become available as aheadof-print articles. The first medium through which we have started doing this is Twitter. As part of this, IJSPP soon will start by asking submitting authors to also indicate their Twitter handle (user name) as part of the submission process. This step will enable us to link IJSPP tweets to the author(s) of the paper. In addition, we are working on creating a "practical application" tweet for each paper, which will highlight how the outcomes of a paper can be used or are beneficial in a practical applied way. It is our hope that IJSPP will not only become more visible scientifically but also become more impactful in the application of sport science in sport practice.

\#FollowUsToday @IJSPPJournal!

Jos $J$ de Koning, IJSPP Editor, Vrije Universiteit Amsterdam, the Netherlands

Teun van Erp, IJSPP Social Media Editor and Rob Lamberts, IJSPP Associate Editor,

Stellenbosch University, South Africa

Stephen Cheung, IJSPP Associate Editor, Brock University, Canada

Dionne Noordhof, IJSPP Editorial Assistant, Norwegian University of Science and Technology, Norway

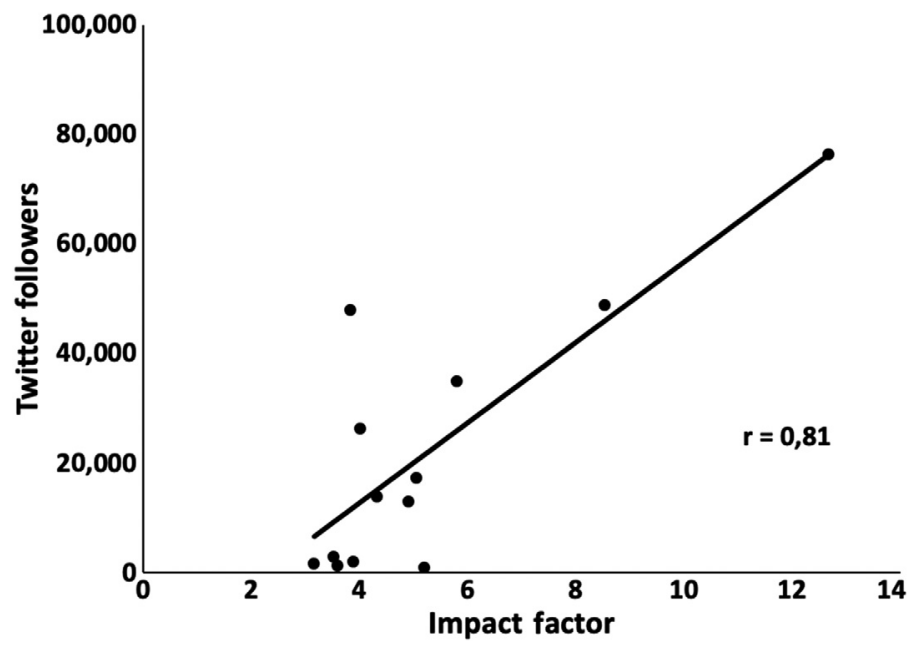

Figure 1 - The relationship between the number of Twitter followers and the impact factor of the top-15 sport-science journals. 


\section{References}

1. Sandbakk $\varnothing$. Let's close the gap between research and practice to discover new land together! Int J Sports Physiol Perform. 2018;13(8): 961. doi:10.1123/ijspp.2018-0550
2. Coutts AJ. Building a bridge between research and practice-the importance of the practical application. Int J Sports Physiol Perform. 2020;15(4):449. doi:10.1123/ijspp.2020-0143

3. Bucheit M. Whom do we publish for? ourselves or others? Int J Sports Physiol Perform. 2020;15(8):1057-1058. doi:10.1123/ijspp.2020-0656 